\title{
Exact semiclassical evolutions in relativistic and non-relativistic scalar quantum mechanics and quantum cosmology
}

\author{
Ali Mostafazadeh ' \\ Theoretical Physics Institute, University of Alberta. Edmonton, Alberta, T6G $2 J /$ Canada \\ College of Arts and Sciences. Kof University, Istinye, 80860 Istanbul, Turkey ${ }^{2}$
}

Received 30 January 1997; revised 29 July 1997; accepted 8 September 1997

\begin{abstract}
The necessary and sufficient conditions for the exactness of the semiclassical approximation for the solution of the Schrödinger and Klein-Gordon equations are obtained. It is shown that the existence of an exact semiclassical solution of the Schrödinger equation determines both the semiclassical wave function and the interaction potential uniquely up to the choice of the boundary conditions. This result also holds for the Klein-Gordon equation. Its implications for the solution of the Wheeler-DeWitt equation for the FRW scalar field minisuperspace models are discussed. In particular, exact semiclassical solutions of the Wheeler-DeWitt equation for the case of massless scalar field and exponential matter potentials are constructed. The existence of exact semiclassical solutions for polynomial matter potentials of the form $\lambda \phi^{2 p}$ is also analyzed. It is shown that for $p=1,2$ and 3, right-going semiclassical solutions do not exist. A generalized semiclassical perturbation expansion is also developed which is quite different from the traditional $\hbar$ and $M_{p}^{-1}$ expansions. (c) 1998 Published by Elsevier Science B.V.
\end{abstract}

PACS: 03.65.-Sq; 04.20.Jb; 04.60.+n; 11.10.Qr

Keywords: Semiclassical approximation; Minisuperspace Wheeler-DeWitt equation

\section{Introduction}

The semiclassical or WKB approximation [1] is usually discussed in textbooks on non-relativistic quantum mechanics in the context of stationary states, i.e. the determina-

\footnotetext{
' E-mail: alimos@phys.ualberta.ca and amostafazadeh@ku.edu.tr.

${ }^{2}$ Current address.
} 
tion of the energy eigenvalues and eigenfunctions [2]. This approximation can also be used to obtain approximate and in some cases exact solutions of the dynamical problem, i.e. the full Schrödinger equation. To the best of my knowledge, however, the utility of the semiclassical approximation in obtaining exact solutions of the Schrödinger equation has not been fully explored.

The same seems to be the case for the relativistic quantum mechanics. The importance of the semiclassical approximation in the relativistic case is probably best appreciated in quantum cosmology [3,4], specifically, in the analysis of the Wheeler-DeWitt equation which is essentially a Klein-Gordon equation on a superspace [5].

In more general terms, the semiclassical approximation is usually viewed as an approximation scheme in which one neglects all but the first term in an asymptotic perturbation expansion of the solution of a linear differential equation. Typical examples of such an asymptotic expansion are the loop expansions of quantum mechanics and field theory where the perturbation parameter is the Planck constant ${ }^{3} \hbar$ [2]. In the context of quantum cosmology the relevant perturbation parameter is the gravitational coupling constant (or the inverse of the Planck mass $M_{p}$ ) [6-8]. Usually, these perturbation expansions are singular and it is difficult, if not impossible, to obtain their precise structure.

There is a more universal alternative for defining the semiclassical approximation where the validity of the approximation is not linked with the values of the physical constants but determined by the properties of the wave function. In this approach one uses the polar representation of the wave function

$$
\psi(\boldsymbol{x} ; t)=R(\boldsymbol{x} ; t) e^{i S(\boldsymbol{x} ; t) / \hbar},
$$

and obtains two coupled non-linear differential equations for the amplitude $R$ and the phase (angle) $S$ of $\psi$ by substituting (1) in the dynamical equation. As is demonstrated for the Schrödinger and Klein-Gordon equations in Sections 2 and 3 below, there emerges a quantity called the quantum potential $Q$ which controls the coupling of these two equations. In other words, if $Q$ which depends only on $R$ happens to be negligible, then one of the equations decouples from the other. The decoupled equation which only involves $S$ turns out to satisfy a Hamilton-Jacobi equation. Thus, for $Q=0, S$ can be identified with the classical action function of the corresponding classical theory. This observation is originally due to Bohm [9]. It provides the basis for the de Broglie-Bohm causal or ontological interpretation of quantum mechanics [10]. The latter has recently been applied to problems of quantum cosmology by several authors [11].

The idea of the quantum potential leads to a precise criterion for the validity of the semiclassical approximation, namely the condition $Q \approx 0$. More precisely, one has the following:

\footnotetext{
${ }^{3}$ Here I have assumed that the kinetic term in the Lagrangian does not involve a coupling constant. For example in non-relativistic quantum mechanics in a Euclidean space, this corresponds to the case where the mass $m$ of the particle is set to unity. Otherwise, the perturbation parameter for the loop expansion is $\hbar / \sqrt{m}$.
} 
Definition. A wave function is said to be semiclassical if the corresponding quantum potential vanishes identically.

Note that the quantum potential $Q$ is determined by the amplitude $R$ of the wave function. Thus, the validity of the semiclassical approximation has nothing to do with the value of the physical constants which are fixed by nature. It is solely decided on the basis of the particular form of the wave function. This in turn depends on the interaction potential and the boundary conditions of the problem.

The purpose of this article is to derive the necessary and sufficient conditions on the interaction potential and the boundary conditions under which the dynamical equations, namely the Schrödinger equation in the non-relativistic case and Klein-Gordon equation in the relativistic case, are exactly solved by a semiclassical wave function. This is done in Sections 2 and 3. Here the problem of the classification of all potentials which allow for exact semiclassical wave functions is solved. Section 4 includes a detailed analysis of the $(1+1)$-dimensional Klein-Gordon equation. The results are then applied in Section 5 for the study of solutions of the Wheeler-DeWitt equation for FRW scalar field minisuperspace models. Here several exact semiclassical solutions are constructed. In Section 6, the ideas and the results of the preceding sections are used to develop a novel semiclassical perturbation theory. The latter yields the semiclassical approximation in the zeroth order of the perturbation theory. The higher-order corrections are shown to satisfy linear differential equations with vanishing boundary conditions. In this way the information about the boundary conditions of the original problem is included in the zeroth order (semiclassical) terms and the definition of the perturbation potential. Section 7 is devoted to a brief examination of the relationship between the semiclassical approximation scheme developed in this paper and the conventional semiclassical approximation. Here, it is shown that the latter can indeed be viewed as a special case of the former. Section 8 presents the concluding remarks.

\section{Non-relativistic QM: Schrödinger equation}

Consider the Schrödinger equation

$$
\begin{aligned}
i \hbar \frac{d}{d t} \psi(t) & =\hat{H} \psi(t), \quad \psi(0)=\psi_{0}, \\
\hat{H} & =\frac{1}{2 m}[\hat{\boldsymbol{p}}-\boldsymbol{A}(\hat{\boldsymbol{x}} ; t)]^{2}+V(\hat{\boldsymbol{x}} ; t),
\end{aligned}
$$

where $\psi$ is a state vector represented in the position representation by the complex scalar wave function $\langle\boldsymbol{x} \mid \psi(t)\rangle=\psi(\boldsymbol{x} ; t), \boldsymbol{A}$ is an electromagnetic vector potential, and $V$ is a scalar interaction potential.

Inserting Eq. (1) in the Schrödinger Eq. (2) and making use of $\langle\boldsymbol{x}| \hat{\boldsymbol{p}}=-i \hbar \nabla(\boldsymbol{x} \mid$, one obtains

$$
\partial_{t} S(\boldsymbol{x} ; t)+H\left(\boldsymbol{x}, \boldsymbol{p}_{*} ; t\right)+Q(\boldsymbol{x} ; t)=0,
$$




$$
\partial_{t} \rho(\boldsymbol{x} ; t)+\nabla \cdot \boldsymbol{J}(\boldsymbol{x} ; t)=0,
$$

where $H=H(x, p ; t)$ is the classical Hamiltonian, $\rho:=R^{2}, p_{*}:=\nabla S, Q:=-\hbar^{2} \nabla^{2} R$ $/(2 m R)$ is the quantum potential, and $\boldsymbol{J}:=\rho \boldsymbol{v}_{*}$ with $\boldsymbol{v}_{*}:=\left(\boldsymbol{p}_{*}-\boldsymbol{A}\right) / m$, is the probability current.

Eq. (3) is the quantum analog of the Hamilton-Jacobi equation,

$$
\partial_{t} S(\boldsymbol{x} ; t)+H\left(\boldsymbol{x}, p_{*} ; t\right)=0,
$$

of the classical mechanics [12]. It differs from the latter because of the presence of the quantum potential $Q$. Eq. (4) is the continuity equation signifying the conservation of the probabilities.

According to the above definition, the semiclassical or WKB approximation provides the exact solution of the Schrödinger equation, if and only if in addition to Eqs. (3) and (4), one has

$$
Q:=\frac{-\hbar^{2}}{2 m} \frac{\nabla^{2} R}{R}=0 \quad \Longleftrightarrow \quad \nabla^{2} R=0,
$$

i.e. $R$ is a solution of the Laplace equation. In this case, Eq. (3) reduces to the Hamilton-Jacobi equation (5). Therefore, the necessary and sufficient conditions for the exactness of the semiclassical approximation are (5), (4), and (6). These equations may be solved for the three unknown functions $R, S$ and $V$.

Eq. (6) does not involve time derivatives. It is really a constraint equation which can be independently solved. Solving Eq. (6) and substituting the result in (4), one finds a first-order equation for $v_{*}$ which in turn yields $p_{*}$. This leads to another first-order differential equation for $S$. The potential $V$ is then obtained by solving the latter equation and substituting the result in Eq. (5).

There is an alternative way of solving the continuity equation (4) which involves writing it explicitly in terms of $S$, namely, considering the solution of

$$
\nabla \cdot\left(R^{2} \nabla S\right)=R f
$$

where $f:=-2 m \partial_{t} R+2 \nabla R \cdot A+R \nabla \cdot A$. Now, let us define $\widetilde{S}:=R S$. Then, it is an easy exercise to show that $\widetilde{S}$ is the solution of the following Poisson equation:

$$
\nabla^{2} \widetilde{S}=f
$$

Here I have used in addition to Eq. (7) the constraint equation (6). Hence, $S$ is given by a solution of the Poisson equation (8) divided by a (non-zero) solution of the Laplace equation (6). Note that both of these equations are second-order elliptic differential equations with well-posed boundary-value problems. Thus, $R, S$ and consequently $V$ are uniquely determined by the boundary conditions. This solves the problem of the classification of all non-relativistic (scalar) quantum systems with an exact semiclassical solution for the Schrödinger equation by relating the latter to the boundary conditions of the Laplace and Poisson equations. It is also important to note that these boundary 
conditions may in general depend on time, which appears in the corresponding equations as a parameter.

In order to demonstrate the utility of these findings in concrete terms, I shall next consider the case where the classical configuration space is one dimensional. Here one can pursue according to the former approach of integrating the continuity equation (4) by first solving for $v_{*}$.

\subsection{One-dimensional configuration spaces}

Consider a quantum system whose configuration space is the interval $\left[x_{1}, x_{2}\right] \subset \mathbb{R}$, and let the boundary conditions on the solution of the Schrödinger equation (2) be given by $\psi\left(x_{1}, t\right)=\psi_{1}(t)=: R_{1}(t) \exp \left[i S_{1}(t) / \hbar\right], \psi\left(x_{2}, t\right)=\psi_{2}(t)=: R_{2}(t) \exp \left[i S_{2}(t) / \hbar\right]$. In this case, Eq. (6) becomes $\partial_{x}^{2} R=0$ which yields

$$
R=a(t) x+b(t)
$$

where

$$
a(t)=\frac{R_{1}(t)-R_{2}(t)}{x_{1}-x_{2}}, \quad b(t)=\frac{x_{1} R_{1}(t)-x_{2} R_{2}(t)}{x_{1}-x_{2}} .
$$

Substituting (9) in Eq. (4) and integrating the resulting differential equation, one obtains

$$
\begin{aligned}
v_{*} & =\frac{1}{(a x+b)^{2}}\left[-\frac{2}{3} a \partial_{t} a x^{3}-\left(a \partial_{t} b+b \partial_{t} a\right) x^{2}-2 b \partial_{t} b x+c\right], \\
S & =d+m\left[-\left(\frac{2 a \partial_{t} a}{3}\right) I_{3}-\left(a \partial_{t} b+b \partial_{t} a\right) I_{2}-2 b \partial_{t} b I_{1}+c I_{0}\right]+\int A d x,
\end{aligned}
$$

where $c=c(t)$ and $d=d(t)$ are functions of time determined by equating the right-hand side of (12) with $S_{1}$ and $S_{2}$ at $x=x_{1}$ and $x=x_{2}$, respectively, and

$$
I_{k}:=\int \frac{x^{k} d x}{(a x+b)^{2}}, \quad k=0,1,2,3 .
$$

More explicitly, one has

$$
\begin{aligned}
& I_{0}=\frac{-1}{a(a x+b)}, \quad I_{1}=\frac{1}{a^{2}}\left[\ln |a x+b|+\frac{b}{a x+b}\right], \\
& I_{2}=\frac{1}{a^{3}}\left[a x+b-2 b \ln |a x+b|+\frac{b^{2}}{a x+b}\right], \\
& I_{3}=\frac{1}{a^{4}}\left[\frac{1}{2}(a x+b)^{2}-3 b(a x+b)+3 b^{2} \ln |a x+b|+\frac{b^{3}}{a x+b}\right] .
\end{aligned}
$$

The potential is then obtained using Eq. (5), namely

$$
V=-\partial_{t} S-\frac{m}{2} v_{*}^{2}
$$


It has the following general form:

$$
V=C_{-1}(t) \ln [a(t) x+b(t)]+\frac{\sum_{\ell=0}^{6} C_{\ell}(t) x^{\ell}}{[a(t) x+b(t)]^{4}}-\int \partial_{t} A(x ; t) d x,
$$

where $C_{\ell}$, with $\ell=-1,0,1, \ldots, 6$, depend on $a, b, c$, and $d$.

In view of the above analysis, one can reach the following conclusions:

- The condition of the exactness of the semiclassical approximation together with the boundary conditions determine both the semiclassical wave function and the potential uniquely.

- For $x_{1} \rightarrow-\infty$ and $x_{2} \rightarrow \infty$, i.e. for a particle in $\mathbb{R}$, a smooth semiclassical wave function is not normalizable. It corresponds to a scattering state.

- More general exact semiclassical wave functions may be obtained by allowing a countable number of discontinuities. The effect of these discontinuities is to divide the interval $\left[x_{1}, x_{2}\right]$ into a collection of subintervals in the interior of which the wave function and potential are given by the above expressions. The boundary conditions corresponding to each subinterval can be chosen freely. They determine the global structure of the wave function and the potential which can now be more complicated. This observation can also be used to devise an approximation scheme for the solution of the Schrödinger equation, by approximating the solution by a locally semiclassical one.

Next, let us consider the following special cases:

(1) Constant boundary conditions: $\partial_{t} \psi_{1}=\partial_{t} \psi_{2}=0$ with $R_{1} \neq R_{2}$ In this case, $a$ and $b$ do not depend on time. This simplifies the above formulae considerably. One has

$$
\begin{aligned}
& v_{*}=\frac{c(t)}{(a x+b)^{2}}, \quad S=d(t)-\frac{m c(t)}{a(a x+b)}+\int A(x) d x, \\
& V=-\partial_{t} d(t)+\frac{m \partial_{t} c(t)}{a(a x+b)}-\frac{m c(t)^{2}}{2(a x+b)^{4}}-\int \partial_{t} A(x) d x,
\end{aligned}
$$

where

$$
\begin{gathered}
c=\frac{\left(S_{2}-S_{1}\right)+\left(\gamma_{1}-\gamma_{2}\right)}{\zeta_{1}-\zeta_{2}}, \quad d=\frac{\left(\zeta_{1} S_{2}-\zeta_{2} S_{1}\right)+\left(\zeta_{2} \gamma_{1}-\zeta_{1} \gamma_{2}\right)}{\zeta_{1}-\zeta_{2}}, \\
\gamma_{i}:=\gamma\left(x_{i}\right), \quad \gamma(x):=\int A(x) d x, \quad \zeta_{i}:=\frac{m}{a\left(a x_{i}+b\right)}, \quad i=1,2 .
\end{gathered}
$$

In particular, for $A=0, \gamma_{i}$ vanish and $c$ and $d$ are constant. This leads to a further simplification of Eqs. (14) and (15) and yields

$$
S=d-\frac{m c}{a(a x+b)}, \quad V=-\frac{m v_{*}^{2}}{2}=-\frac{m c^{2}}{2(a x+b)^{4}} .
$$

In this case both the potential and the action function turn out to be time independent. This corresponds to a semiclassical zero-energy eigenfunction. 
(2) Amplitude-periodic boundary conditions, i.e. $R_{1}(t)=R_{2}(t)$

In this case, $a=0$ and $R=b(t)$. Then, a similar analysis leads to

$$
\begin{aligned}
v_{*}= & \frac{-2 b \partial_{t} b x+c}{b^{2}}, \quad S=d+m\left[-\left(\partial_{t} \ln b\right) x^{2}+\frac{c}{b^{2}} x\right]+\int A d x \\
V= & -\partial_{t} d-m\left[-\left(\partial_{t}^{2} \ln b\right) x^{2}+\partial_{t}\left(c / b^{2}\right) x\right]-\frac{m}{2}\left[\frac{-2 b \partial_{t} b x+c}{b^{2}}\right]^{2} \\
& -\int \partial_{t} A d x
\end{aligned}
$$

Here, $c$ and $d$ depend on the phases $S_{i}$ of $\psi_{i}$ according to

$$
c=\frac{\Sigma_{2}(t)-\Sigma_{1}(t)}{\alpha_{2}(t)-\alpha_{1}(t)}, \quad d=\frac{\Sigma_{1}(t) \alpha_{2}(t)-\Sigma_{2}(t) \alpha_{1}(t)}{\alpha_{2}(t)-\alpha_{1}(t)},
$$

where

$$
\alpha_{i}:=\frac{m x_{i}}{b^{2}}, \quad \Sigma_{i}:=S_{i}-\gamma_{i}+m\left(\partial_{t} \ln b\right) x_{i}^{2} .
$$

Note also that in this case, for $A=$ constant, the potential $V$ is at most a quadratic polynomial in $x$. For, $b=R_{1}=R_{2}=$ constant, $V$ is either a first-order or a zeroth-order polynomial in $x$. For example, for $b=1, S_{i}=\omega_{i} t$, with $\omega_{i}$ being real constants, and $A=0$, one has

$$
\begin{aligned}
c & =\frac{\left(\omega_{2}-\omega_{1}\right) t}{m\left(x_{2}-x_{1}\right)}, \quad d=\frac{\left(\omega_{1} x_{2}-\omega_{2} x_{1}\right) t}{x_{2}-x_{1}}, \\
S & =\left(\frac{t}{x_{2}-x_{1}}\right)\left[\left(\omega_{1} x_{2}-\omega_{2} x_{1}\right)+\left(\omega_{2}-\omega_{1}\right) x\right], \\
V & =\left(\frac{-1}{x_{2}-x_{1}}\right)\left[\left(\omega_{1} x_{2}-\omega_{2} x_{1}\right)+\left(\omega_{2}-\omega_{1}\right) x\right]-\frac{1}{2 m}\left[\frac{\left(\omega_{2}-\omega_{1}\right) t}{\left(x_{2}-x_{1}\right)}\right]^{2} .
\end{aligned}
$$

Another interesting case is when $b=e^{\omega t / 2}$ for some constant $\omega$. Then, the potential is a quadratic polynomial in $x$ with the coefficient of the quadratic term being a constant, namely, $-m \omega^{2} / 2$. In the latter case if the phases $S_{1}$ and $S_{2}$ and the vector potential $A$ are also time independent, then so are $c$ and $d$. Therefore, one has a time-independent quadratic potential, namely

$$
V=-\frac{m}{2}(\omega x+c)^{2}
$$

\subsection{Multi-dimensional configuration spaces}

For an $n$-dimensional configuration space, with $n>1$, the classification of the exact semiclassical wave functions and the corresponding potentials is more involved. This is mainly because in this case the constraint equation $(6)$ is the $n$-dimensional Laplace equation $\nabla^{2} R=0$. 
In Cartesian coordinates, one can use the method of separation of variables to express $R$ as a sum of the basic solutions

$$
\prod_{i=1}^{n}\left\{a_{i}(t) \mathcal{S}\left[\kappa_{i}(t) x^{i}\right]+b_{i}(t) \mathcal{C}\left[\kappa_{i}(t) x^{i}\right]\right\},
$$

where $\left(x^{1}, \ldots, x^{n}\right):=\boldsymbol{x}, \kappa_{i}, a_{i}$, and $b_{i}$ are real functions of time which are determined by the boundary conditions on the wave function, the $\kappa_{i}$ satisfy

$$
\sum_{i=1}^{n} \eta_{i} \kappa_{i}^{2}(t)=0
$$

with $\eta_{i}= \pm 1$, and $\mathcal{S}$ (respectively $\mathcal{C}$ ) stands for either of sin or sinh (respectively cos or cosh) depending on whether $\eta_{i}=-1$ or +1 , respectively. Clearly, one can choose one of the $\eta_{i}$ 's positive and the others negative.

Having found the expression for $R$, one then proceeds by integrating the continuity equation (4) which yields $S$. In view of the above analysis, $S=\widetilde{S} / R$, where $\widetilde{S}$ is a solution of the Poisson equation (8). Using the well-known Green function methods [13] of solving the Poisson equation, one may express $S$ in an integral form. For example, if the configuration space is $\mathbb{R}^{3}$, then

$$
\begin{aligned}
S(x ; t)= & \frac{1}{R(\boldsymbol{x} ; t)}\left\{\widetilde{S}_{0}(x ; t)+\frac{1}{2 \pi} \int d x^{\prime 3}\right. \\
& \left.\times\left[\frac{m \partial_{t} R\left(\boldsymbol{x}^{\prime} ; t\right)-\nabla R\left(\boldsymbol{x}^{\prime} ; t\right) \cdot A\left(\boldsymbol{x}^{\prime} ; t\right)-\frac{R\left(x^{\prime} ; t\right)}{2} \nabla \cdot A\left(\boldsymbol{x}^{\prime} ; t\right)}{\left|\boldsymbol{x}-\boldsymbol{x}^{\prime}\right|}\right]\right\},
\end{aligned}
$$

where $\widetilde{S}_{0}$ is a solution of the Laplace equation determined by the boundary conditions.

Similarly to the one-dimensional case, in the case that the configuration space is $\mathbb{R}^{n}$, a smooth semiclassical wave function cannot be normalized. More generally, it cannot vanish at infinity, nor can it be localized. This is a direct consequence of Eq. (19).

The main difference with the one-dimensional case is that here one has a much richer structure as far as the general form of the wave function and the potential is concerned. Unfortunately, since without knowing the specific form of the boundary conditions one cannot express $R$ and $S$ in a closed form, an explicit classification of the semiclassical wave functions and the corresponding potentials for $n>1$ is not available. Nevertheless, it is evident that by choosing the boundary conditions appropriately one can obtain a large variety of potentials.

In order to demonstrate the validity of this claim, I shall next concentrate on the special cases where the amplitude of the semiclassical wave function is independent of $\boldsymbol{x}$. A simple example of this is a particle in a cubical cavity of side length $L$ with the boundary conditions

$$
\left.\psi\right|_{\partial}=N(t) e^{i S_{\partial}(x ; t) / \hbar}
$$


where the symbol $\partial$ stands for the boundary of the cavity. In this case, $R=N(t)$ is the unique solution of the constraint equation (6), and $\nabla R=0$. This reduces Eq. (7) to the simple Poisson equation

$$
\nabla^{2} S=-2 m \partial_{t} \ln N
$$

where I have chosen the Coulomb gauge so that $\nabla \cdot \boldsymbol{A}=0$. Note that the source term on the right-hand side of Eq. (21) only depends on time. Hence, one can define $\check{S}:=S+m\left[\partial_{t} \ln N\right]|\boldsymbol{x}|^{2}$ and reduce this equation to the Laplace equation

$$
\nabla^{2} \check{S}=0 \text {. }
$$

Since the set of solutions of the Laplace equation are in one to one correspondence with the set of boundary conditions which is a very large function space, one obtains a large class of potentials.

Next, consider the following simple subcases.

(1) $\check{S}_{\partial}=\left.\mathcal{K}(t) \cdot \boldsymbol{x}\right|_{\partial}$, where $\mathcal{K}$ is an $\boldsymbol{x}$-independent vector-valued function of time. Then, $\breve{S}=\mathcal{K}(t) \cdot \boldsymbol{x}$ clearly satisfies the Laplace equation (22) and one has

$$
\begin{aligned}
S= & \mathcal{K}(t) \cdot \boldsymbol{x}-m\left[\partial_{t} \ln N(t)\right]|\boldsymbol{x}|^{2}, \\
V= & m\left[\partial_{t}^{2} \ln N(t)\right]|\boldsymbol{x}|^{2}-\partial_{t} \mathcal{K}(t) \cdot \boldsymbol{x} \\
& -\frac{1}{2 m}\left|2 m\left[\partial_{t} \ln N(t)\right] \boldsymbol{x}+\boldsymbol{A}(\boldsymbol{x} ; t)-\mathcal{K}(t)\right|^{2} .
\end{aligned}
$$

This case is the multi-dimensional analog of Example (2) of Section 2.1.

(2) Consider the case $n=2$, i.e. a square with boundaries $x^{1}=: x=0, L$ and $x^{2}=: y=$ $0, L$, and boundary conditions: $\breve{S}=0$ for $x=0, L, y=0$, and $\breve{S}=\alpha(t) \sin (\pi x / L)$ for $y=L$. Then, one can easily show that the solution of the Laplace equation (22) is given by

$$
\check{S}=\frac{\alpha(t) \sin (\pi x / L) \sinh (\pi y / L)}{\sinh \pi} .
$$

Hence, one has

$$
\begin{aligned}
S= & \frac{\alpha(t) \sin (\pi x / L) \sinh (\pi y / L)}{\sinh \pi}-m\left[\partial_{t} \ln N(t)\right]|x|^{2}, \\
V= & m\left[\partial_{t}^{2} \ln N(t)\right]|x|^{2}-\frac{\partial_{t} \alpha(t) \sin (\pi x / L) \sinh (\pi y / L)}{\sinh \pi}-\frac{\alpha^{2}(t)}{2 m \sinh ^{2} \pi} \\
& \times\left\{\left[\frac{\pi}{L} \cos (\pi x / L) \sinh (\pi y / L)-2 m\left[\partial_{t} \ln N(t)\right] x-\frac{\sinh (\pi) A_{x}}{\alpha(t)}\right]^{2}\right. \\
& \left.+\left[\frac{\pi}{L} \sin (\pi x / L) \cosh (\pi y / L)-2 m\left[\partial_{t} \ln N(t)\right] y-\frac{\sinh (\pi) A_{y}}{\alpha(t)}\right]^{2}\right\}
\end{aligned}
$$

These relations show that unlike the one-dimensional case, here the wave function and the potential can be quite complicated. 
So far, I have only considered cases for which $\nabla R=0$. This is precisely the condition demanded in the traditional semiclassical approximation. It is usually argued that the semiclassical approximation is valid if the amplitude $R$ of the wave function is a slowly varying function of $\boldsymbol{x}$. As is clear from the above analysis, this is only a sufficient condition, not a necessary one. There is an infinite number of examples where $R$ is a rapidly changing function of $\boldsymbol{x}$ but the semiclassical approximation is not only valid, but it yields the exact result. Specific examples can be constructed by simply choosing $R$ to be a rapidly changing solution of the Laplace equation. For instance, consider a quantum system with the geometry of the preceding example, but the boundary conditions which lead to

(3) $R(x, y ; t)=R(x, y)=N_{0} \sin (\ell \pi x / L) \sinh (\ell \pi y / L)$ and $S(x, y ; t)=S(x, y)=$ $\mathcal{K} \cdot \boldsymbol{x} / R$, where $N_{0}$ and $\ell$ are real and integer constants, respectively, and $\mathcal{K}$ is a constant vector. One can easily check that $R$ and $S$ satisfy Eqs. (6) and (7). However,

$$
|\nabla R|=\left|\frac{\ell N \pi}{L} \cos \left(\frac{\ell \pi x}{L}\right) \cosh \left(\frac{\ell \pi y}{L}\right)\right| \sqrt{\tan ^{2}\left(\frac{\ell \pi x}{L}\right)+\tanh ^{2}\left(\frac{\ell \pi y}{L}\right)}
$$

can be made arbitrarily large by choosing large values for $\ell$, i.e. $R$ is not a slowly varying function of $x$ and $y$. Note also that in this case both $R$ and $S$ are time independent. Thus, the wave function describes a zero-energy eigenfunction of the time-independent potential

$$
V=-\frac{(\nabla S)^{2}}{2 m}=\frac{-1}{2 m R^{2}}\left[\mathcal{K}^{2}+\frac{(\mathcal{K} \cdot \boldsymbol{x})^{2}(\nabla R)^{2}}{R^{2}}-\frac{2(\mathcal{K} \cdot \boldsymbol{x})(\mathcal{K} \cdot \nabla R)}{R}\right] .
$$

This example clearly shows how the present analysis generalizes the results of the traditional semiclassical approach to quantum mechanics.

\section{Relativistic QM: Klein-Gordon equation}

Consider the Klein-Gordon equation

$$
\left[\left(\partial^{\mu}-A^{\mu}\right)\left(\partial_{\mu}-A_{\mu}\right)-V(x)\right] \psi(x)=0,
$$

where $A_{\mu}$ are components of an electromagnetic gauge field, $V$ is a scalar interaction potential (including the mass term in the massive case), and $x$ stands for the four vector $\left(x^{\mu}\right)$. In the following, I shall follow the relativists' convention for the Minkowski metric, namely, $\left(\eta_{\mu \nu}\right)=\operatorname{diag}(-1,1, \ldots, 1)$, and set $c=\hbar=1$.

In the polar representation (1), the Klein-Gordon equation is written as

$$
\begin{array}{r}
\left(\partial^{\mu} S-A^{\mu}\right)\left(\partial_{\mu} S-A_{\mu}\right)+V+Q=0, \\
\partial_{\mu} J^{\mu}=0,
\end{array}
$$


where $Q:=-\partial^{\mu} \partial_{\mu} R / R$ is the quantum potential and $J^{\mu}:=\rho\left(\partial^{\mu} S-A^{\mu}\right)$, with $\rho:=R^{2}$, is the conserved current. Again, Eq. (24) is the quantum analog of the Hamilton-Jacobi equation for a classical relativistic particle

$$
\left(\partial^{\mu} S-A^{\mu}\right)\left(\partial_{\mu} S-A_{\mu}\right)+V=0,
$$

and Eq. (25) is the continuity equation associated with the charge conservation.

A semiclassical Klein-Gordon field is defined by the condition

$$
Q:=-\frac{\partial^{\mu} \partial_{\mu} R}{R}=0 \quad \Longleftrightarrow \quad \partial^{\mu} \partial_{\mu} R=0 .
$$

Therefore, the semiclassical or WKB approximation is exact if and only if the relations (27), (26), and (25) are satisfied. As in the non-relativistic case, these three equations may be used to determine the three unknown real functions $R, S$, and $V$. This is done by first solving Eq. (27) which is already decoupled from the other two. This is a wave equation for $R$. Its general solution is given by a linear combination of the functions of the form

$$
W_{\hat{k}}=W_{\hat{k}}\left(x^{0}-\boldsymbol{x} \cdot \hat{\boldsymbol{k}}\right) \text {, }
$$

where $x=\left(x^{0}, \boldsymbol{x}\right)$ belongs to the $(n+1)$-dimensional Minkowski space $\mathcal{M}^{n+1}$ or a subset of $\mathcal{M}^{n+1}$, and $\hat{\boldsymbol{k}}$ is a unit $n$-vector defining the null wave $(n+1)$-vector $k=k_{0}(1, \hat{\boldsymbol{k}})$. A simple choice for $W_{\hat{\boldsymbol{k}}}$ which is essentially motivated by the Fourier analysis of the wave equation is the plane waves exp $i\left(k_{0} x^{0}-\boldsymbol{x} \cdot \boldsymbol{k}\right)$. Once the $W_{\hat{k}}$ are chosen, the solution of Eq. (27) reduces to the determination of the coefficients of $W_{\hat{k}}$ in the expansion of $R$.

Next, one substitutes the expression for $R$ in the continuity equation (25) and integrates the resulting equation. The basic strategy is similar to the non-relativistic case. In terms of $S$ the continuity equation (25) takes the form

$$
\partial^{\mu}\left(R^{2} \partial_{\mu} S\right)=R F
$$

where $F:=2 \partial_{\mu} R A^{\mu}+R \partial_{\mu} A^{\mu}$. Eq. (29) is the analog of Eq. (7). It can further be simplified by defining $\widetilde{S}:=R S$. This together with Eqs. (27) and (29) leads to

$$
\partial^{\mu} \partial_{\mu} \widetilde{S}=F
$$

i.e. $S=\widetilde{S} / R$, where $\widetilde{S}$ is a solution of the inhomogeneous wave equation (30). Having found $R$ and $S$, one can use the Hamilton-Jacobi equation (26) to determine the form of the potential.

There is a very important difference between the relativistic and non-relativistic cases. Here the condition of the exactness of the semiclassical approximation leads to two second-order hyperbolic equations, namely (27) and (30), whereas in the nonrelativistic case one has two elliptic equations. One knows from the theory of hyperbolic differential equations that the boundary-value problem for such equations is not generally well posed, i.e. for arbitrary boundary conditions, a solution may or may not exist and 
if it does, it may not be unique. The well-posed problem for a hyperbolic equation such as the wave equations (27) and (30) is the initial-value problem. In general, for given initial data on a Cauchy hypersurface one can solve these equations and determine $R$, $S$, and $V$ uniquely. One of the consequences of the hyperbolicity of (27) is that unlike the non-relativistic case, here $R$ and therefore the semiclassical wave function can be localized. In particular, one can form a coherent wave packet which approximates the behavior of a classical particle.

Restricting to the case where $R$ is a constant and adopting the Lorentz gauge $\partial^{\mu} A_{\mu}=$ 0 , one can reduce Eq. (29) to a (homogeneous) wave equation for $S$,

$$
\partial^{\mu} \partial_{\mu} S=0
$$

The general solution of this equation is also given as a linear combination of functions of the form (28). This is sufficient to conclude that even for this special case $S$ and consequently $V$ can be quite complicated. This shows that there is a large class of potentials which allow exact semiclassical solutions of the Klein-Gordon equation. These potentials and the corresponding semiclassical Klein-Gordon fields depend in a crucial way on the boundary conditions. ${ }^{4}$ This is especially important in quantum cosmology where there is an ongoing controversy regarding the choice of the boundary conditions for the wave function of the universe and also the form of the potential in the Wheeler-DeWitt equation. In particular, for the FRW scalar field minisuperspace models $[3,4]$, the Wheeler-DeWitt equation is precisely a $(1+1)$-dimensional KleinGordon equation of the form (23). For these models the form of the potential is directly linked with the phenomenon of inflation [14,3,4]. On the other hand, most if not all the physical predictions which one hopes to derive from such models are relevant to the regions of the minisuperspace where the wave function is semiclassical. The results of this paper indicate that at least one can rule out the cases where the existence of a semiclassical solution (for some region of the minisuperspace) is inconsistent with the form of the potential (in that region). This together with the requirements imposed by inflation may be helpful in improving our understanding of quantum cosmology. This motivates a closer analysis of the Klein-Gordon equation in the $(1+1)$-dimensional Minkowski space.

\section{Klein-Gordon equation in $(1+1)$ dimensions}

If the configuration space is the $(1+1)$-dimensional Minkowski space $\mathcal{M}^{2}$, then the general solution of the wave equations (27) and (30) can be written in terms of four real-valued functions $R_{ \pm}$and $\widetilde{S}_{ \pm}[15]$

$$
R(x, t)=R_{+}(x+t)+R_{-}(x-t),
$$

\footnotetext{
${ }^{4}$ Here and in what follows, "boundary conditions" means "initial", "boundary", or "mixed initial-boundary conditions" for which there exists at least one solution.
} 


$$
\widetilde{S}(x, t)=\widetilde{S}_{+}(x+t)+\widetilde{S}_{-}(x-t)+\int d x^{\prime} d t^{\prime} G\left(x, t ; x^{\prime}, t^{\prime}\right) F\left(x^{\prime}, t^{\prime}\right),
$$

where $F$ is the same as the one appearing in Eq. (29) and $G$ is the appropriate Green function for the one-dimensional wave equation [15]. The latter can be constructed out of the advanced and retarded Green functions given by $G^{ \pm}\left(x, t ; x^{\prime}, t^{\prime}\right):=\left[\theta\left(\left|x-x^{\prime}\right| \pm\right.\right.$ $\left.\left.\left(t-t^{\prime}\right)\right)-1\right] / 2$, where + and - label the advanced and retarded Green functions, respectively, and $\theta$ is the step function, $\theta(z)=1$, if $z>0 ; \theta(z)=0$, if $z<0$. The usual choice in typical physical applications is the retarded Green function $G^{-}$which marks a particular direction of time. Note that if the electromagnetic potential $A_{\mu}$ is absent, $F=0$, the last term in Eq. (33) drops, and there is no need for a Green function, in particular, a direction of time. Finally, in view of $S=\widetilde{S} / R$ one has

$$
\begin{aligned}
S(x, t)= & \frac{1}{R_{+}(x+t)+R_{-}(x-t)} \\
& \times\left[\widetilde{S}_{+}(x+t)+\widetilde{S}_{-}(x-t)+\int d x^{\prime} d t^{\prime} G\left(x, t ; x^{\prime}, t^{\prime}\right) F\left(x^{\prime}, t^{\prime}\right)\right] .
\end{aligned}
$$

Eqs. (32) and (33) together with Eq. (26) show that in general the exact semiclassical wave functions and the corresponding potentials are classified by the set $\mathcal{C}^{4}:=$ $\left\{\left(R_{ \pm}, \widetilde{S}_{ \pm}\right)\right\}$, where $\mathcal{C}$ is the set of real-valued functions of a single real variable.

Let us next concentrate on the case where there is no electromagnetic interaction. Then, in view of Eq. (26) the potential has the general form

$$
\begin{aligned}
V & =\frac{-4\left[\widetilde{S}_{+}^{\prime} \widetilde{S}_{-}^{\prime}+S^{2} R_{+}^{\prime} R_{-}^{\prime}-S\left(R_{+}^{\prime} \widetilde{S}_{-}^{\prime}+R_{-}^{\prime} \widetilde{S}_{+}^{\prime}\right)\right]}{\left(R_{+}+R_{-}\right)^{2}} \\
& =-4\left(\frac{\widetilde{S}_{+}^{\prime}}{\widetilde{S}_{+}+\widetilde{S}_{-}}-\frac{R_{+}^{\prime}}{R_{+}+R_{-}}\right)\left(\frac{\widetilde{S}_{-}^{\prime}}{\widetilde{S}_{+}+\widetilde{S}_{-}}-\frac{R_{-}^{\prime}}{R_{+}+R_{-}}\right) S^{2} \\
& =: \mathcal{F}\left[R_{ \pm}, \widetilde{S}_{ \pm}\right],
\end{aligned}
$$

where $R_{ \pm}=R_{ \pm}(x \pm t), \widetilde{S}_{ \pm}=\widetilde{S}_{ \pm}(x \pm t)$, a prime means first derivative of the corresponding function,

$$
S=\frac{\tilde{S}_{+}+\widetilde{S}_{-}}{R_{+}+R_{-}},
$$

and the function(al) $\mathcal{F}$ is defined for future use. Eq. (34) is obtained by substituting (32) and (35) in the Hamilton-Jacobi equation (26).

Next, consider the simple case $R_{ \pm}=\frac{1}{2}$. Then, $S=\widetilde{S}_{+}+\widetilde{S}_{-}$and $V=-4 \widetilde{S}_{+}^{\prime} \widetilde{S}_{-}^{\prime}$. In particular, one has the following interesting examples:

(1) $S$ is linear in time $t: \widetilde{S}_{ \pm}=\omega_{ \pm}(x \pm t) / 2$ for some constants $\omega_{ \pm}$

In this case, $V=-\omega_{+} \omega_{-}=$constant. This includes the case of a free KleinGordon field of mass $\mu=\sqrt{-\omega_{+} \omega_{-}}$, since in this case $V=\mu^{2}$.

(2) $S$ is quadratic in $t$ and $x: \widetilde{S}_{ \pm}=\nu_{ \pm}(x \pm t)^{2} / 2$ for some constants $\nu_{ \pm}$

In this case, one obtains a quadratic potential of the form $V=4 \nu_{+} \nu_{-}\left(t^{2}-x^{2}\right)$. This 
corresponds to a Klein-Gordon field with the time-dependent mass $\mu=2 \sqrt{\nu_{+} \nu_{-}} t$ and a quadratic interaction potential. The non-relativistic limit of this case is a time-dependent harmonic oscillator with imaginary frequency.

(3) $S$ is a linear combination of exponential functions: $\widetilde{S}_{ \pm}=\nu_{ \pm} e^{\omega_{ \pm}(x \pm t)}$

In this case, one has an exponential potential, $V=V_{0} e^{\left(\omega_{+}+\omega_{-}\right) x+\left(\omega_{+}-\omega_{-}\right) t}$, where $V_{0}:=-4 \omega_{+} \omega_{-} \nu_{+} \nu_{-}$. Clearly, by choosing $\omega_{-}= \pm \omega_{+}=: \pm \omega$, one obtains exponential potentials which depend only on $t$ or $x$, namely $V=V_{0} e^{2 \omega x}$ and $V=V_{0} e^{2 \omega t}$, respectively.

These examples can also be described in the framework of the traditional semiclassical approach, since $R$ is chosen to be unity. Similarly to the non-relativistic case, in order to demonstrate the generality of the present analysis, one must consider the cases where $R$ is a rapidly changing function of $t$ and $x$, but the semiclassical approximation is nevertheless exact. Again typical examples can be constructed starting from a rapidly changing solution of the wave Eq. (27). For instance, consider the case

(4) $R=R_{0} e^{\omega(x-t)}$ and $S=S_{0} e^{-2 \omega x}$

Then one can check that Eqs. (27) and (29) are satisfied and the corresponding semiclassical wave function is an exact solution of the Klein-Gordon equation defined by the time-independent potential $V=-4 \omega^{2} S_{0}^{2} e^{-4 \omega x}$.

Eq. (34) provides a classification of the potentials for which an exact semiclassical solution of the $(1+1)$-dimensional Klein-Gordon equation, with $A_{\mu}=0$, exists. In practice, however, it is the potential which is given, not the wave function. Hence, a more interesting question is whether for a given potential $V=V(x, t)$ there is a set of boundary conditions which yields an exact solution of the Klein-Gordon equation. One can alternatively ask whether a given potential belongs to the image of the function $\mathcal{F}$ defined in Eq. (34).

In order to answer these questions, I shall first consider a special class of boundary conditions, namely $R_{+}=0$. For this class one can show that $\mathcal{F}$ is not onto and the set of potentials from Eq. (34), with $R_{+}=0$, forms a small subset of all possible potentials. To see this, let us first substitute $R_{+}=0$ in (34). The resulting equation may then be viewed as a differential equation for $\tilde{S}_{-}$, while $R_{-}$and $\widetilde{S}_{+}$are treated as undetermined functions. This leads to

$$
\widetilde{S}_{-}^{\prime}+\left(-\frac{R_{-}^{\prime}}{R_{-}}\right) \tilde{S}_{-}+\frac{R_{-}^{2}}{4}\left[\frac{V}{{\widetilde{S_{+}^{\prime}}}_{-}}-\frac{4 R_{-}^{\prime} \widetilde{S}_{+}}{R_{-}^{3}}\right]=0
$$

which is a consistent first-order ordinary linear differential equation for $\tilde{S}_{-}$provided that the bracket on its left-hand side depends only on $x-t$. This puts an strong restriction on the form of the allowed potentials. Namely, the potential must be of the form

$$
V(x, t)=\mathcal{X}(x-t){\widetilde{S_{+}}}_{+}(x+t)+\mathcal{Y}(x-t) \widetilde{S}_{+}(x+t){\widetilde{S_{+}}}_{+}(x+t),
$$

where $\mathcal{X}$ is an arbitrary real-valued function and $\mathcal{Y}:=4 R_{-}^{\prime} / R_{-}^{3}$. 
Proposition 1. Let $u:=x-t$ and $v:=x+t$ be null coordinates in $\mathcal{M}^{2}$ and $V: \mathcal{M}^{2} \rightarrow \mathbb{R}$ be an analytic function at $(0,0) \in \mathcal{M}^{2}$. Then, a necessary condition for $V$ to satisfy Eq. (37), for some functions $\mathcal{X}: \mathbb{R} \rightarrow \mathbb{R}, \mathcal{Y}: \mathbb{R} \rightarrow \mathbb{R}$, and $\tilde{S}_{+}: \mathbb{R} \rightarrow \mathbb{R}$, is that the coefficients $V_{j n}$ in the power series expansion $\sum_{j, n=0}^{\infty} V_{j n} u^{j} v^{n}$ of $V$ must satisfy one of the following two relations:

$$
\begin{aligned}
& V_{j n}=\frac{\left(V_{k_{1} m_{1}} V_{j m_{1}}-V_{k_{1} m_{2}} V_{j m_{2}}\right) V_{k_{2} n}+\left(V_{k_{2} m_{2}} V_{j m_{2}}-V_{k_{2} m_{1}} V_{j m_{1}}\right) V_{k_{1} n}}{V_{k_{1} m_{1}} V_{k_{2} m_{2}}-V_{k_{1} m_{2}} V_{k_{2} m_{1}}}, \\
& V_{j n}=\frac{V_{j m_{1}} V_{k_{1} n}}{V_{k_{1} m_{1}}},
\end{aligned}
$$

with $\left(j, k_{1}, k_{2}\right)$ and $\left(n, m_{1}, m_{2}\right)$ being triplets of mutually different arbitrary non-negative integers.

Proof. Substitute the power series expansions

$$
\begin{aligned}
& V=V(u, v)=: \sum_{j, n=0}^{\infty} V_{j n} u^{j} v^{n}, \quad \mathcal{X}=\mathcal{X}(u)=: \sum_{j=0}^{\infty} \mathcal{X}_{j} u^{j}, \\
& \mathcal{Y}=\mathcal{Y}(u)=: \sum_{j=0}^{\infty} \mathcal{Y}_{j} u^{j}, \quad \widetilde{S}_{+}=\widetilde{S}_{+}(v)=: \sum_{n=0}^{\infty} S_{n} v^{n}
\end{aligned}
$$

in Eq. (37). This leads to

$$
\begin{aligned}
V_{j n} & =(n+1) S_{n+1} \mathcal{X}_{j}+\mathcal{Y}_{j} \sum_{m=0}^{n}(n-m+1) S_{m} S_{n-m_{1}} \\
& =(n+1) S_{n+1} \mathcal{Z}_{j}+\mathcal{Y}_{j} \sum_{m=0}^{n-1}(n-m) S_{m+1} S_{n-m},
\end{aligned}
$$

where $\mathcal{Z}_{j}:=\mathcal{X}_{j}+S_{0} \mathcal{Y}_{j}$. Next, solve for the sum on the right-hand side of (42). The result is

$$
\sum_{m=0}^{n-1}(n-m) S_{m+1} S_{n-m}=\frac{1}{\mathcal{Y}_{j}}\left[V_{j n}-(n+1) S_{n+1} \mathcal{Z}_{j}\right] .
$$

Clearly the left-hand side of this equation is independent of $j$. Hence, its right-hand side must also be independent of $j$. Writing the right-hand side for two different values of $j$ and equating the results, one has

$$
S_{n}=\frac{1}{n}\left(\frac{\mathcal{X}_{j}}{\mathcal{Y}_{j}}-\frac{\mathcal{X}_{k}}{\mathcal{Y}_{k}}\right)^{-1}\left(\frac{V_{j, n-1}}{\mathcal{Y}_{j}}-\frac{V_{k, n-1}}{\mathcal{Y}_{k}}\right), \quad \forall j \neq k .
$$

Next let us express $S_{n}$ using two different values of $k$, say $k_{1}$ and $k_{2}$. Equating the two expressions and simplifying the result, one obtains

$$
\mathcal{X}_{j}=\frac{\mathcal{Y}_{j}}{\mathcal{Y}_{k_{2}}}\left(\mathcal{X}_{k_{2}}+\left(\mathcal{Y}_{k_{1}} \mathcal{X}_{k_{2}}-\mathcal{Y}_{k_{2}} \mathcal{X}_{k_{1}}\right)\left[\frac{\mathcal{Y}_{k_{2}} V_{j n}-\mathcal{Y}_{j} V_{k_{2} n}}{\mathcal{Y}_{k_{1}} \mathcal{Y}_{j} V_{k_{2} n}-\mathcal{Y}_{k_{2}} \mathcal{Y}_{j} V_{k_{1} n}}\right]\right) \text {, }
$$


Since $n$ does not appear in this equation except in the square bracket, the content of the square bracket must be independent of $n$. This argument may be used to determine $\mathcal{Y}_{j}$ by equating the square bracket on the right-hand side of (44) with its value for $n=m_{1}$. This leads to

$$
\mathcal{Y}_{j}=\mathcal{Y}_{k_{2}} \mathcal{W}_{j n}
$$

where

$$
\mathcal{W}_{j n}:=\frac{\left(c V_{k_{2} m_{1}}-V_{k_{1} m_{1}}\right) V_{j n}-\left(c V_{k_{2} n}-V_{k_{1} n}\right) V_{j m_{1}}}{V_{k_{2} m_{1}} V_{k_{1} n}-V_{k_{1} m_{l}} V_{k_{2} n}},
$$

and $c:=\mathcal{Y}_{k_{1}} / \mathcal{Y}_{k_{2}}$. Once again, $\mathcal{W}_{j n}=\mathcal{Y}_{j} / \mathcal{Y}_{k_{2}}$ must be independent of $n$, i.e. for all $m$ and $n, \mathcal{W}_{j n}=\mathcal{W}_{j m}$. In particular $\mathcal{W}_{j n}=\mathcal{W}_{j m_{2}}$, where $m_{2}$ is some arbitrarily chosen fixed non-negative integer. This equation may be used to express $V_{j n}$ in terms of $V_{j m_{1}}, V_{j m_{2}}, V_{k_{1} n}, V_{k_{2} n}$, and $c$, namely

$$
\begin{aligned}
V_{j n}= & {\left[\frac{c V_{k_{2} n}-V_{k_{1} n}}{c V_{k_{2} m_{1}}-V_{k_{1} m_{1}}}-\left(\frac{c V_{k_{2} m_{2}}-V_{k_{1} m_{2}}}{c V_{k_{2} m_{1}}-V_{k_{1} m_{1}}}\right)\left(\frac{V_{k_{2} m_{1}} V_{k_{1} n}-V_{k_{1} m_{1}} V_{k_{2} n}}{V_{k_{2} m_{1}} V_{k_{1} m_{2}}-V_{k_{1} m_{1}} V_{k_{2} m_{2}}}\right)\right] V_{j m_{1}} } \\
& +\left[\frac{V_{k_{2} m_{1}} V_{k_{1} n}-V_{k_{1} m_{1}} V_{k_{2} n}}{V_{k_{2} m_{1}} V_{k_{1} m_{2}}-V_{k_{1} m_{1}} V_{k_{2} m_{2}}}\right] V_{j m_{2}} .
\end{aligned}
$$

Next, consider the following two possibilities:

(I) $c \neq V_{k_{1} m_{1}} / V_{k_{2} m_{1}}$ :

Then, the right-hand side of (47) does not actually depend on $c$. In this case, one finds

$$
V_{j n}=\frac{\left(V_{k_{1} m_{1}} V_{j m_{1}}-V_{k_{1} m_{2}} V_{j m_{2}}\right) V_{k_{2} n}+\left(V_{k_{2} m_{2}} V_{j m_{2}}-V_{k_{2} m_{1}} V_{j m_{1}}\right) V_{k_{1} n}}{V_{k_{1} m_{1}} V_{k_{2} m_{2}}-V_{k_{1} m_{2}} V_{k_{2} m_{1}}}
$$

which is just Eq. (38).

(II) $c=V_{k_{1} m_{1}} / V_{k_{2} m_{1}}$ :

Then, according to the definition of $c$, i.e. $c:=\mathcal{Y}_{k_{1}} / \mathcal{Y}_{k_{2}}$ and Eq. (43) either all $S_{n}$ vanish - this corresponds to the trivial case $V_{j n}=0-$ or $\mathcal{X}_{j} / \mathcal{Y}_{j}=\mathcal{X}_{k} / \mathcal{Y}_{k}$, i.e. the ratio $\mathcal{X}_{j} / \mathcal{Y}_{j}$ does not depend on $j$. The latter implies $\mathcal{X}=\eta \mathcal{Y}$ for some constant $\eta$. Moreover, in this case $V_{j n}$ must satisfy

$$
V_{j n}=\frac{V_{j m_{1}} V_{k_{1} n}}{V_{k_{1} m_{1}}}
$$

which is just Eq. (39).

Note that in the latter case, Eq. (39) together with $\mathcal{X}=\eta \mathcal{Y}$ and Eqs. (42) and (37) lead to

$$
\begin{aligned}
V_{j n} & =v_{n} \mathcal{Y}_{j}, \quad v_{n}:=\eta(n+1) S_{n+1}+\sum_{m=0}^{n}(n-m+1) S_{m} S_{n-m}, \\
V(x, t) & =\left[\eta+\widetilde{S}_{+}(x+t)\right] \widetilde{S}_{+}^{\prime}(x+t) \mathcal{Y}(x-t) .
\end{aligned}
$$


Now, substituting Eq. (49) in Eq. (36), one can easily show that indeed $\widetilde{S}_{+}$drops out of this equation and one obtains $\widetilde{S}_{-}=\eta$. Therefore, the exact semiclassical wave function $\psi=R e^{i S}$ is given by $R=R_{-}$and $S=\left(\eta+\widetilde{S}_{+}\right) / R_{-}$. For example, consider choosing

(5) $\mathcal{Y}=\mu_{-} e^{\omega_{-}(x-t)}$ and $\left(\eta+\widetilde{S}_{+}\right) \widetilde{S}_{+}^{\prime}=\mu_{+} e^{\omega_{+}(x+t)}$ for some constants $\mu_{ \pm}$and $\omega_{ \pm}$: Then, one has $V=\mu_{+} \mu_{-} e^{\left(\omega_{+}+\omega_{-}\right) x} e^{\left(\omega_{+}-\omega_{-}\right) t}$. In particular, for $\omega_{+}=-\omega_{-}=: \omega$, the potential depends only on $t$. In this case, one has

$$
\begin{aligned}
& V=\mu_{+} \mu_{-} e^{2 \omega t}, \quad R=\sqrt{\frac{\nu_{-}+\mu_{-} e^{-\omega(x-t)}}{2 \omega}}, \\
& S= \pm 2 \sqrt{\frac{\mu_{+} e^{\omega x}+\nu_{+} e^{-\omega t}}{\mu_{-} e^{-\omega x}+\nu_{-} e^{-\omega t}}},
\end{aligned}
$$

where $\nu_{ \pm}$are also constants. The appearance of the square roots in these equations is an indication that for certain choices of $\mu_{ \pm}$and $\nu_{ \pm}$either $R$ or $S$ can become imaginary in some regions of the Minkowski space. Since $R$ and $S$ are assumed to be real, such a semiclassical solution does not exist in these regions.

This is another example of a case where the amplitude of an exact semiclassical solution is not a slowly varying function of its arguments.

A simple consequence of Proposition 1 is

Corollary. The potentials of the form (37) which are analytic at $(x=0, t=0)$ form a proper subset of the set of all potentials, i.e. for an arbitrary potential which is analytic at $(x=0, t=0)$, an exact semiclassical solution of the $(1+1)$-dimensional Klein-Gordon equation (23), with $R_{+}=0$ and $A_{\mu}=0$, may not exist.

Eqs. (38) and (39) provide a useful criterion for finding out whether a given potential allows for an exact semiclassical solution with $R_{+}=0$ or not. If the result is positive, then Eqs. (43)-(45) may be used to determine $R_{-}$and $\widetilde{S}_{+}$. These are then used to integrate Eq. (36) which yields $\widetilde{S}_{-}$and consequently the wave function $\psi=R_{-} \exp \left[i\left(\widetilde{S}_{-}+\right.\right.$ $\left.\left.\widehat{S}_{+}\right) / R_{-}\right]$.

Proposition 1 only applies to the cases where $R_{+}=0$. One might try to employ a similar method to treat the more general case, where $R_{+}$is also an undetermined function. For this purpose, one must first write Eq. (34) as a polynomial equation in $V, R_{ \pm}, \tilde{S}_{ \pm}$and their derivatives and substitute the power series expansions of these functions in the resulting expression. This leads to an infinite system of very complicated coupled non-linear algebraic equations for the coefficients of $R_{ \pm}, \widetilde{S}_{ \pm}$whose analytic solution has not been possible. Although a similar proof is lacking for the most general case, further inspection of Eq. (34) suggests that this equation also restricts the form of the potential. Hence, in general for an arbitrary potential an exact semiclassical solution of the Klein-Gordon equation does not exist. 


\section{Exact semiclassical wave functions of the universe}

Consider the Wheeler-DeWitt equation for the closed FRW cosmological model coupled to a scalar field $\phi[3,4]$,

$$
\left[-\partial_{\alpha}^{2}+\partial_{\phi}^{2}+e^{4 \alpha}-e^{6 \alpha} \mathcal{V}(\phi)\right] \psi=0
$$

where $\alpha:=\ln a, a$ is the scale factor of the FRW model, and $\mathcal{V}$ is the matter field potential. Here, the cosmological constant is assumed to vanish, the usual factor ordering prescription [3,4] is adopted, and the natural units in which the Planck mass is set to unity is used. Clearly, this is a Klein-Gordon equation in $(1+1)$-dimensional Minkowski (minisuper) space with potential

$$
V=-e^{4 \alpha}+e^{6 \alpha} \mathcal{V}(\phi),
$$

where $(\alpha, \phi)$ play the role of $(t, x)$.

The solutions of Eq. (51) have been studied mostly for the massless $(\mathcal{V}=0)$ and massive $\left(\mathcal{V}=m^{2} \phi^{2}\right)$ scalar fields in the literature [16,17,19,3,20,4,21]. This is done by making use of different approximation schemes except for the rather trivial and much less interesting massless case for which the exact solution is known $[22,19]$. The approximate solutions of Eq. (51) are usually developed by making particular assumptions for the boundary conditions, semiclassicality of the solution, or restricting to particular regions of the minisuperspace in which the Wheeler-DeWitt equation (51) simplifies.

In view of the developments reported in the preceding sections, the assumption of the exactness of the semiclassical approximation provides a direct link between the choice of the boundary conditions and the form of the potential. This is done through the function $\mathcal{F}$ defined by (34) which can be viewed as a function from the set of boundary conditions to the set of potentials $V$. In Section 4, I have shown for the case $R_{+}=0$ that this function is not onto, i.e. there are potentials which do not admit exact semiclassical solutions. Here, however, one is interested in a special class of potentials, namely those of the form (52). Hence, the relevant problem is to find the intersection $\mathcal{U}$ of the image of $\mathcal{F}$ and the set of potentials of the form (52). One can easily show that $\mathcal{U}$ is not empty. For example, the massless case, where $V$ is an exponential function of $\alpha$, can be easily put in the form (34), i.e. it belongs to $\mathcal{U}$. In fact, two possible choices for $R_{ \pm}$and $\widetilde{S}_{ \pm}$which lead to this potential were already given in Examples (3) and (5) of Section 4 (with $\omega=2$ ). The following are non-trivial examples of the potentials of the form (52) which also belong to $\mathcal{U}$. They are obtained by setting $R_{+}=0$ and making simple choices for the functions $\mathcal{X}, \mathcal{Y}$, and $\widetilde{S}_{+}$of Eq. (37) so that Eq. (52) is also satisfied. This together with Eq. (36) yields $\widetilde{S}_{-}$.

(1) $\mathcal{V}=\lambda e^{2 \phi}$ with $\lambda>0$

In this case, the choices $\mathcal{X}=-e^{-2(\phi-\alpha)}, R_{-}=2 e^{\phi-\alpha} / \sqrt{\lambda}$, and $\widetilde{S}_{+}=e^{2(\phi+\alpha)} / 2$ satisfy Eq. (37). The solution of (36) then leads to $\widetilde{S}_{-}=c e^{\phi-\alpha}+1 / \lambda$, where $c$ is a constant. The exact semiclassical solution is given by 


$$
R=\frac{2 e^{\phi-\alpha}}{\sqrt{\lambda}}, \quad S=\sqrt{\lambda}\left(e^{\phi+3 \alpha}+\frac{2 e^{\alpha-\phi}}{\lambda}+2 c\right) .
$$

(2) $\mathcal{V}=\lambda e^{-4 \phi}$ with arbitrary $\lambda$

In this case, one has $\mathcal{X}=\lambda e^{-5(\phi-\alpha)} / c_{1}, R_{-}=2 c_{1}\left[c_{2}-e^{-2(\phi-\alpha)}\right]^{-1 / 2}, \tilde{S}_{+}=$ $c_{1} e^{\phi+\alpha}$, and

$$
\begin{aligned}
\tilde{S}_{-}= & -\frac{\lambda c_{1}}{8}\left[2 e^{-3(\phi-\alpha)}+3 c_{2} e^{-(\phi-\alpha)}\right. \\
& \left.+3 c_{2}^{2} e^{\phi-\alpha}\left(\frac{\tan ^{-1} \sqrt{c_{2} e^{2(\phi-\alpha)}-1}+c_{3}}{\sqrt{c_{2} e^{2(\phi-\alpha)}-1}}\right)\right],
\end{aligned}
$$

where $c_{1}, c_{2}$ and $c_{3}$ are constants. The exact semiclassical solution is therefore given by

$$
\begin{aligned}
R= & \frac{2 c_{1}}{\sqrt{c_{2}-e^{-2(\phi-\alpha)}}}, \\
S= & \left(\frac{e^{\phi+\alpha}}{2}-\frac{\lambda\left[2 e^{-3(\phi-\alpha)}+3 c_{2} e^{-(\phi-\alpha)}\right]}{16}\right) \sqrt{c_{2}-e^{-2(\phi-\alpha)}} \\
& -\frac{3 \lambda c_{2}^{2}}{16}\left(\tan ^{-1} \sqrt{c_{2} e^{2(\phi-\alpha)}-1}+c_{3}\right) .
\end{aligned}
$$

Note that in this case, there is always a region of the minisuperspace defined by $e^{\phi-\alpha}<c_{2}^{-1 / 2}$ where a semiclassical solution does not exist.

The next logical step is to explore the existence of exact semiclassical solutions of the Wheeler-DeWitt equation with matter potentials of the form $\mathcal{V}=\lambda \phi^{2 \prime}$. These are among the potentials which lead to inflationary classical solutions [14]. The simplest case is that of a massive scalar field, i.e. $\lambda=m^{2}, p=1$. In the remainder of this section, I shall restrict to the semiclassical solutions with $R_{+}=0$. The existence of this type of solutions can be easily decided using Eqs. (38) and (39). One simply needs to compute the coefficients $V_{j n}$ of (40) and check whether they satisfy one of these equations. A simple calculation shows that for $p=1,2,3$, none of these equations are satisfied. Hence, the matter potentials $\lambda \phi^{2}, \lambda \phi^{4}$, and $\lambda \phi^{6}$ do not admit exact "right-going" $\left(R_{+}=0\right)$ semiclassical solutions. This is done by choosing the integers $j, n, k_{1}, k_{2}, m_{1}$, and $m_{2}$ in such a way that both Eqs. (38) and (39) fail. In order to demonstrate this, let us denote the right-hand sides of Eqs. (38) and (39) by $V_{j n}^{(1)}$ and $V_{j n}^{(2)}$, respectively. Then,

- for $p=1$ and $k_{1}=m_{1}=0, k_{2}=m_{2}=1$, one finds

$$
\begin{array}{ll}
V_{22}=-4+\frac{9 \lambda}{4}, & V_{22}^{(1)}=-4+\frac{13 \lambda}{8}-\frac{\lambda^{2}}{16},
\end{array}
$$

- for $p=2$ and $k_{1}=m_{1}=0, k_{2}=m_{2}=2$, one finds 


$$
\begin{array}{ll}
V_{31}=\frac{8}{3}+\frac{\lambda}{4}, & V_{31}^{(1)}=V_{31}^{(2)}=\frac{8}{3}, \\
V_{33}=\frac{16}{9}-\frac{9 \lambda}{8}, & V_{33}^{(1)}=\frac{16}{9}+\frac{3 \lambda}{8}, \quad V_{33}^{(2)}=\frac{16}{9},
\end{array}
$$

- for $p=3$ and $k_{1}=m_{1}=0, k_{2}=m_{2}=3$, one finds

$$
V_{24}=-\frac{4}{3}+\frac{15 \lambda}{64}, \quad V_{24}^{(1)}=V_{24}^{(2)}=-\frac{4}{3} \text {. }
$$

Note that for $\lambda=0$, i.e. the massless case, the values of $V_{j n}$ in the above list match the values of $V_{j n}^{(1)}$ and $V_{j n}^{(2)}$. This is in agreement with the fact that for the massless case one does in fact have right-going exact semiclassical solutions. Eq. (50) with $(t, x) \rightarrow(\alpha, \phi), \omega=2$, and $\mu_{-}=-1 / \mu_{+}$, provides a concrete example.

Furthermore, one knows from the studies of the inflationary cosmological models that for the polynomial matter potentials $\lambda \phi^{2 p}$ the coupling constant $\lambda$ must be a very small number. For example for the massive case, where $p=1$ and $\lambda=m^{2}$, these theories predict $m \approx 10^{-6}$, i.e. $\lambda \approx 10^{-12}$ [14]. Thus, although there are no rightgoing semiclassical solutions for $p=1,2,3$, at least for small values of $u=\phi-\alpha$ and $v=\phi+\alpha$, where one can neglect forth- and higher-order terms in the power series expansion of $V$, the semiclassical approximation seems to be reliable.

The phrase semiclassical approximation is used in quantum cosmology in a very crude way. One usually makes additional assumptions such as the adiabaticity of the evolution $[17,18]$ to reduce the situation to the one-dimensional quantum mechanical case. In this way, one is able to express the condition of the validity of the semiclassical approximation as a simple limitation on the range of values of the matter potential [4]. The consistency of these assumptions with the validity of the semiclassical approximation is either left unchecked or a set of conditions is imposed which render the scheme consistent. These conditions are usually sufficient conditions, not necessary. Hence, in general they may be too restrictive. The situation is very similar to restricting the exact semiclassical wave functions to those with slowly varying amplitudes. As shown in the preceding sections, this is an absolutely unnecessary restriction. The approach pursued in the article also allows for a precise definition of a more general semiclassical approximation where the solution to the dynamical equations is approximated with the general semiclassical wave functions introduced in Section 1. This will be discussed next.

\section{Semiclassical perturbation theory}

Let us first define a semiclassical potential $V_{0}$ to be a potential which corresponds to an exact semiclassical solution of the dynamical equation. In view of the results of Sections 2 and 3, the set of semiclassical potentials is in fact much larger than one usually expects. This suggests a generalized notion of semiclassical expansion and in particular semiclassical approximation, which corresponds to a perturbation theory around the semiclassical potentials. 
Let $V$ be an arbitrary potential, $\psi=R e^{i S / \hbar}$ be the solution of the dynamical equation, and $\epsilon \in \mathbb{R}$ be a perturbation parameter. Then, the semiclassical perturbation theory corresponds to

$$
\begin{array}{ll}
V=V_{0}+\delta V, & \delta V=\epsilon V_{\mathrm{p}}, \\
R=R_{0}+\delta R, & \delta R=\sum_{\ell=1}^{\infty} \epsilon^{\ell} R_{\ell}, \\
S=S_{0}+\delta S, & \delta S=\sum_{\ell=1}^{\infty} \epsilon^{\ell} S_{\ell},
\end{array}
$$

where $V_{0}$ and $\psi_{0}=R_{0} e^{i S_{0} / \hbar}$ are the semiclassical potential and wave function defined by the boundary conditions of the problem. Substituting Eqs. (53)-(55) in the dynamical equations and treating $\epsilon$ as an independent parameter, one obtains an infinite family of equations which can be iteratively solved to yield $R_{\ell}$ and $S_{\ell}$. In particular, the equations obtained in the $\ell$ th order are two linear coupled differential equations in $R_{\ell}$ and $S_{\ell}$ with vanishing boundary conditions. This is because the original boundary conditions are already imposed in the determination of $R_{0}$ and $S_{0}$.

Eqs. (54) and (55) yield what one might call a semiclassical perturbation expansion. Note that they are not power series in $\hbar$ but in the perturbation parameter $\epsilon$. The zerothorder terms correspond to the semiclassical wave function. Thus, the semiclassical approximation is defined by $R \approx R_{0}$ and $S \approx S_{0}$. Note also that the semiclassical wave function $\psi_{0}$ and potential $V_{0}$ are uniquely determined by the boundary conditions. The choice of the perturbation parameter is, however, made by the physics of the problem. A typical example of a perturbation parameter is the coupling constant $\lambda$ of the preceding section.

Let us next list the equations governing the first and second-order terms in the semiclassical perturbation expansion.

- Non-relativistic QM: Schrödinger equation

First-order (post-semiclassical) corrections, i.e. equations determining $R_{1}$ and $S_{1}$,

$$
\begin{array}{r}
\partial_{t} S_{1}+\frac{1}{m}\left(\nabla S_{0}-A\right) \cdot \nabla S_{1}+V_{p}-\frac{\hbar^{2} \nabla^{2} R_{1}}{2 m R_{0}}=0, \\
2 \partial_{t}\left(R_{0} R_{1}\right)+\frac{1}{m} \nabla \cdot\left[R_{0}^{2} \nabla S_{1}+2 R_{0} R_{1}\left(\nabla S_{0}-A\right)\right]=0 .
\end{array}
$$

Second-order corrections, i.e. equations determining $R_{2}$ and $S_{2}$,

$$
\begin{gathered}
\partial_{t} S_{2}+\frac{1}{2 m}\left[\left(\nabla S_{1}\right)^{2}+2\left(\nabla S_{0}-A\right) \cdot \nabla S_{2}\right] \\
-\frac{1}{2 m}\left(\frac{\nabla^{2} R_{2}}{R_{0}}-\frac{R_{1} \nabla^{2} R_{1}}{R_{0}^{2}}\right)=0,
\end{gathered}
$$




$$
\begin{aligned}
& 2 \partial_{t}\left(R_{0} R_{2}\right)+\partial_{t} R_{1}^{2} \\
& \quad+\frac{1}{m} \nabla \cdot\left[\left(R_{1}^{2}+2 R_{0} R_{2}\right)\left(\nabla S_{0}-A\right)+2 R_{0} R_{1} \nabla S_{1}+R_{0}^{2} \nabla S_{2}\right]=0 .
\end{aligned}
$$

These equations are obtained by substituting Eqs. (53)-(55) in Eqs. (3) and (4).

- Relativistic QM: Klein-Gordon equation $(c=h=1)$

First-order (post-semiclassical) corrections, i.e. equations determining $R_{1}$ and $S_{1}$,

$$
\begin{aligned}
& 2\left(\partial^{\mu} S_{0}-A^{\mu}\right) \partial_{\mu} S_{1}+V_{\mathrm{p}}+\frac{\partial^{\mu} \partial_{\mu} R_{1}}{R_{0}}=0, \\
& \partial_{\mu}\left[2 R_{0} R_{1}\left(\partial^{\mu} S_{0}-A^{\mu}\right)+R_{0}^{2} \partial^{\mu} S_{1}\right]=0 .
\end{aligned}
$$

Second-order corrections, i.e. equations determining $R_{2}$ and $S_{2}$,

$$
\begin{gathered}
2\left(\partial^{\mu} S_{0}-A^{\mu}\right) \partial_{\mu} S_{2}+\partial^{\mu} S_{1} \partial_{\mu} S_{1}+\frac{\partial^{\mu} \partial_{\mu} R_{2}}{R_{0}}-\frac{R_{1} \partial^{\mu} \partial_{\mu} R_{1}}{R_{0}^{2}}=0, \\
\partial_{\mu}\left[R_{0}^{2} \partial^{\mu} S_{2}+2 R_{0} R_{1} \partial^{\mu} S_{1}+\left(R_{1}^{2}+2 R_{0} R_{2}\right)\left(\partial^{\mu} S_{0}-A^{\mu}\right)\right]=0 .
\end{gathered}
$$

These equations are obtained by substituting Eqs. (53)-(55) in Eqs. (24) and (25). They can be further simplified. For example, consider the first-order equations (56) and (57), and define $p_{0}^{\mu}:=\partial^{\mu} S_{0}-A^{\mu}$ and $T_{ \pm 1}:=R_{1} \pm R_{0} S_{1}$. Then using the fact that $R_{0}$ and $S_{0}$ define a semiclassical wave function, i.e. $\partial_{\mu} \partial^{\mu} R_{0}=0$ and $\partial_{\mu}\left(R_{0}^{2} p_{0}^{\mu}\right)=0$, one can show that Eqs. (56) and (57) are equivalent to

$$
\left[\partial_{\mu} \partial^{\mu} \pm 2 p_{0}^{\mu}\left(\partial_{\mu}-\partial_{\mu} \ln R_{0}\right)\right] T_{ \pm 1}=-R_{0} V_{\mathrm{P}}
$$

These are two separate equations for $T_{ \pm 1}$ whose solution yields $R_{1}$ and $S_{1}$.

These equations appear to be even more difficult to solve than the original dynamical equations. Note, however, that they are to be solved with vanishing boundary conditions. The advantage of this scheme is that the information on the boundary conditions of the original dynamical equation is restored in the definition of $R_{0}$ and $S_{0}$. The higherorder corrections are affected by these boundary conditions only through $R_{0}, S_{0}$ and the perturbation potential $V_{\mathrm{p}}$ which appears in the first order of perturbation. In this sense, the semiclassical perturbation theory has a universal character.

As a concrete example consider the Wheeler-DeWitt equation of Section 5 with a matter potential of the form $\mathcal{V}=\lambda f(\phi)$, where $f$ is some real-valued function. Let the boundary conditions be such that one recovers Example (5) of Section 4 with $\omega=2$, $\mu_{ \pm}=\mp 1, \nu_{-}=0$, and $\nu_{+}=\nu$. Then replacing $(t, x)$ with $(\alpha, \phi),(V, R, S)$ with ( $\left.V_{0}, R_{0}, S_{0}\right)$, and adopting the positive sign in Eqs. (50), one has

$$
V_{0}=-e^{4 \alpha}, \quad R_{0}=\frac{1}{2} e^{-(\phi-\alpha)}, \quad S_{0}=2 \sqrt{\nu e^{2(\phi-\alpha)}-e^{4 \phi}}
$$

These correspond to an approximate semiclassical solution which is an exact solution of the massless case. Clearly, the perturbation potential $V_{\mathrm{P}}$ is given by $e^{6 \alpha} f(\phi)$ and the 
perturbation parameter is $\lambda$. The first-order (post-semiclassical) correction $\left(R_{1}, S_{1}\right)$ to $\left(R_{0}, S_{0}\right)$ is obtained by solving Eq. (58) which takes the form

$$
\left\{-\partial_{\alpha}^{2}+\partial_{\phi}^{2} \pm\left[\xi\left(\partial_{\alpha}-1\right)+\zeta\left(\partial_{\phi}+1\right)\right]\right\} T_{ \pm 1}=-\frac{1}{2} e^{-\phi+7 \alpha} f(\phi)
$$

with

$$
\xi:=-2 \partial_{\alpha} S_{0}=\frac{4 \nu e^{2(\phi-\alpha)}}{\sqrt{\nu e^{2(\phi-\alpha)}-e^{4 \phi}}}, \quad \zeta:=2 \partial_{\phi} S_{0}=\frac{4\left(\nu e^{2(\phi-\alpha)}-2 e^{4 \phi}\right)}{\sqrt{\nu e^{2(\phi-\alpha)}-e^{4 \phi}}} .
$$

Although solving Eq. (60) seems much more difficult than solving the original WheelerDeWitt equation (51), one must recall that these equations are to be solved with vanishing boundary conditions. In this way one can at least devise an efficient numerical scheme which can treat the solution of these and the equations for the higher-order corrections for arbitrary matter potentials.

In general, such a scheme should first compute the semiclassical potential $V_{0}$ and wave function $R_{0} e^{i S_{0}}$ using the boundary conditions. This would yield the perturbation potential $V_{\mathrm{p}}$ (after the perturbation parameter is identified according to the physical characteristics of the problem). Then, it should numerically integrate the equations satisfied by $R_{\ell}$ and $S_{\ell}$ with vanishing boundary conditions.

Finally, let me emphasize that for the Wheeler-DeWitt equation with the massive scalar field, the perturbation parameter is already extremely small, $\lambda=m^{2}=\mathcal{O}\left(10^{-12}\right)$, therefore the first-order corrections provide solutions which are valid up to the order $\lambda^{2}=$ $\mathcal{O}\left(10^{-24}\right)$. This suggests that the domain of the validity of the first-order perturbation theory is indeed quite large.

\section{Relation with the conventional semiclassical approximation}

In the preceding sections, I have outlined a semiclassical approximation scheme which relies on the same basic principle as the conventional semiclassical approximation. ${ }^{5}$ However, there are obvious differences between the two methods, and it seems necessary to investigate their relationship.

Probably, the main difference between the conventional and the present semiclassical approximation is in the role played by the boundary conditions. In the conventional scheme, one does not, a priori, include the information about the boundary conditions. In fact, as I show in the following, the boundary conditions are universally chosen in the conventional scheme. Therefore, for the cases that the original physical problem requires a different set of boundary conditions, the conventional method cannot be applied.

In order to investigate the relationship between the two methods, I shall consider the one-dimensional Schrödinger equation. In particular, let us recall the results of Example (2) of Section 2.1, for the special case where $A=0$ and $b$ and $c$ are constant. Furthermore, suppose that the time dependence of the wave function is given by a

\footnotetext{
${ }^{5}$ This is most evident in its utility of the classical Hamilton-Jacobi equation.
} 
factor $\exp (-i E t / \hbar)$. This would correspond to the evolution of a stationary state of a conservative quantum system.

The condition that the wave function represents a stationary state corresponds to the choice: $d=-E t$ in Eq. (16). This together with $b, c=$ constant lead to

$$
R=b=\text { constant }, \quad S=-E t+p_{*} x, \quad V=E-E_{0}=\text { constant }
$$

where $p_{*}=m c / b^{2}=\partial_{x} S, E_{0}:=m c^{2} /\left(2 b^{4}\right)=p_{*}^{2} /(2 m)$, and use is made of Eqs. (16) and (17). Expressing the constants $b$ and $E_{0}$ in terms of $p_{*}$, one can write Eqs. (61) in the form

$$
\begin{aligned}
p_{*} & = \pm \sqrt{2 m(E-V)}, \\
S & =-E t \pm \sqrt{2 m(E-V)} x, \\
R & =\sqrt{\frac{m c}{p_{*}}}=(m c)^{1 / 2}[2 m(E-V)]^{-1 / 4} .
\end{aligned}
$$

These equations correspond to the case where the semiclassical approximation yields the exact result. As it is seen from Eqs. (61), this only happens for the trivial case where the potential is constant.

In order to obtain the results for the case where the semiclassical approximation is valid as an approximation, one must require the basic equations (5)-(7) to be satisfied approximately. For the case of the above example, this would mean $R \approx$ constant. In this case, if one first solves the Hamilton-Jacobi equation (5), one recovers the familiar result

$$
S=-E t \pm \int \sqrt{2 m[\bar{E}-V(x)]} d x=-E t \pm \int p_{*}(x) d x,
$$

instead of (63). In order to compute $R$, one then substitutes Eq. (65) in Eq. (7). This leads to the same expression for $R$ as given by (64).

Note that in the domain of validity of the (conventional) semiclassical approximation, $R$ and consequently $p_{*}$ are slowly varying functions. Therefore, Eqs. (63) and (65) agree in this domain. Furthermore, it is interesting to see that expressing the condition that the amplitude of the wave function must be a slowly varying function, i.e. $\partial_{x} \rho \approx 0$, in terms of $V$, one arrives at the well-known condition [2]

$$
\frac{\partial_{x} V}{[2 m(E-V)]^{3 / 2}} \approx 0
$$

The above analysis shows that the conventional semiclassical approximation can be viewed as a special case of the method developed in this paper. More precisely, the conventional semiclassical solution corresponds to the case where the boundary conditions are given by

$$
a=0, \quad b, c=\text { constant, } \quad d=-E t,
$$

where $a, b, c$ and $d$ are defined in Section 2.1. In this case the exact semiclassical evolution is only allowed for the trivial case where the potential is constant. The conventional 
semiclassical approximation is valid as an approximation where the basic equations, namely Eqs. (5)-(7), are satisfied approximately.

It must be emphasized that in general the simple boundary conditions which correspond to the conventional semiclassical approximation, namely (66), are not valid, and the conventional scheme cannot be applied. Such situations are, for instance, encountered in the study of non-conservative quantum systems with time-dependent boundary conditions.

The generality of approach pursued in this paper is even more evident for higherdimensional Schrödinger equations. Here one can find examples where the assumption of the conventional method, namely the requirement that the amplitude of a semiclassical wave function is a slowly varying function, fails even for some stationary states. A concrete example of such a case is Example (3) of Section 2.2.

The situation is quite similar for the Klein-Gordon equation. In particular, for the problems where one is interested in a variety of boundary conditions, the semiclassical approximation scheme outlined in this paper is more convenient than the conventional scheme. Typical examples of such systems can be found in quantum cosmology where one is interested in a semiclassical treatment of the Wheeler-DeWitt equation for a variety of boundary conditions.

The precise relationship between the conventional $\hbar$ or $M_{\mathrm{p}}^{-1}$ expansion and the semiclassical perturbation expansion is not clear for the author. For the quantum cosmological problems, such as those of Section 5, the semiclassical perturbation theory seems to yield a natural approximation scheme. Its advantage over the $\hbar$ or $M_{\mathrm{p}}^{-1}$ expansions is again in its treatment of the boundary conditions. This is the main feature which distinguishes the two methods.

\section{Conclusion}

In this article I have tried to demonstrate how the simple observation that the traditional condition of the validity of the semiclassical approximation is only a sufficient condition, can be used to introduce the notions of an exact semiclassical wave function and potential.

I have shown that the semiclassical wave function and potential are both determined by the boundary conditions of the problem uniquely. I have also given the full classification of exact semiclassical wave functions and potentials for the Schrödinger and Klein-Gordon equations in arbitrary dimensions. The analysis of the one-dimensional Schrödinger equation is much simpler than the multi-dimensional case. For the KleinGordon equation in $(1+1)$ dimensions which is directly relevant to the solution of the Wheeler-DeWitt equation for FRW scalar field minisuperspace models, I have explicitly constructed semiclassical wave functions and potentials. For this case, I have also developed a practical criterion for checking whether a given potential allows for a right-going exact semiclassical solution. I have then used this criterion to study the semiclassical solutions of the minisuperspace Wheeler-DeWitt equation. For the polynomial matter 
potentials of the form $\lambda \phi^{2 p}$ with $p=1,2,3$, I have shown that a right-going semiclassical solution of the Wheeler-DeWitt equation does not exist. However, the non-existence proof relies on the non-zero value of the coupling constant $\lambda$ which is expected to be an extremely small number. This motivated the development of a semiclassical perturbation theory which yields the semiclassical approximation as the zeroth-order term in the semiclassical perturbation expansion. The higher-order terms satisfy coupled linear differential equations with vanishing boundary conditions.

The conventional semiclassical evolution turns out to be a special case of the semiclassical evolutions studied in this paper. It corresponds to a simple choice of the boundary conditions. The conventional semiclassical approximation is obtained by requiring that the basic equations, namely Eqs. (5) - (7) be satisfied approximately for these boundary conditions.

An important advantage of the semiclassical approximation developed in this paper is that it allows for arbitrary boundary conditions. This makes the results of this paper directly applicable in the study of the dynamics of quanturn systems with time-dependent boundary conditions, in general, and quantum cosmology in particular.

\section{Acknowledgements}

I would like to thank B. Darian and M. Razavi for helpful discussions. I would also like to acknowledge the financial support of the Killam Foundation of Canada.

\section{References}

[1] J. Liouville, J. Math. 2(16) (1837) 418;

Lord Rayleigh, Proc. R. Soc. London A 86 (1912) 207;

H. Jeffreys, Proc. London Math. Soc. (2) 23 (1923) 428;

G. Wentzel, Z. Phys. 38 (1926) 518;

H. A. Kramers, Z. Phys. 39 (1926) 828;

L. Brillouin, Comtes Rendus 183 (1926) 24;

R.E. Langer, Phys. Rev. 51 (1937) 669.

[2] D. Bohm, Quantum Theory (Prentice-Hall, Englewood Cliffs, NJ, 1951);

Ph.M. Morse and H. Feshbach, Methods of Theoretical Physics, Vol. 2 (McGraw-Hill, New York, 1953);

L.I. Schiff, Quantum Mechanics (McGraw-Hill, New York, 1955);

A. Messiah, Quantum Mechanics, Vol. 1 (North-Holland, Amsterdan, 1961);

R. Bellman, Perturbation Techniques in Mathematics, Physics, and Engineering (Holt, Rinehart and Winston, New York, 1964);

L.D. Landau and E.M. Lifshitz, Quantum Mechanics (Pergamon, Oxford, 1976);

J.J. Sakurai, Modern Quantum Mechanics (Benjamin/Cummings, Menlo Park, CA, 1985);

R. Shankar, Principles of Quantum Mechanics (Plenum, New York, 1994);

B.R. Holstein, Topics in Advanced Quantum Mechanics (Addison-Wesley, Redwood City, CA, 1992).

[3] D.N. Page, Lectures on quantum cosmology, in Gravitation: A Banff Summer Institute, ed. R. Mann and

P. Wesson (World Scientific, Singapore, 1991).

14| D.L. Wiltshire, An Introduction to quantum cosmology, in Cosmology: The Physics of the Universe, Adelaide preprint no ADP-95-11/M28 (World Scientific, Singapore, to appear).

15] B.S. DeWitt, Phys. Rev. 160 (1967) 1113. 
[6] T.P. Singh and T. Padamanabhan, Ann. Phys. 196 (1989) 296;

T. Padamanabhan and T.P. Singh, Class. Quantum. Grav. 7 (1990) 411.

$17 \mid$ C. Keifer, The semiclassical approximation to quantum gravity, in Canonical Gravity: From Classical to Quantum, ed. J. Ehlers and H. Friedrich (Springer, Berlin, 1994).

181 S.P. Kim, Phys. Rev. D 52 (1995) 3382.

19 ] D. Bohm. Phys. Rev. 85 (1952) 166; Phys. Rev. 85 (1952) 180.

$10 \mid$ D. Bohm, Causality and Chance in Modern Physics (Routledge, London, 1957);

D. Bohm and B.J. Hiley, Phys. Rep. 144 (1987) 349;

P. Holland, Phys. Rep. 224 (1993) 95;

D. Bohm and B.J. Hiley, The Undivided Universe (Routledge, London, 1993).

11 J, Kowalski-Glikman and J.C. Vink, Class. Quantum Grav. 7 (1990) 901;

J.C. Vink, Nucl. Phys B 369 (1992) 707;

S.P. de Alwis and D.A. MacIntire, Phys. Rev. D 50 (1994) 5164;

A. Blaut and J. Kowalski-Glikman, Class. Quantum Grav. 13 (1996) 39;

Y.V. Shtanov, Phys. Rev. D 54 (1996) 2584

121 H. Goldstein, Classical Mechanics (Addison-Wesley, Reading, MA 1980).

131 J.D. Jackson, Classical Electrodynamics (Wiley, New York, 1975).

$14 \mid$ A. Linde, Physica Scripta T36 (1991) 30; see also Phys. Lett. B 162 (1985) 281;

B.L. Al'tshuler and A.O. Barvinsky, Phys. Uspekhi 39 (1996) 429;

D.H. Lyth, Models of inflation and the spectral index of the density perturbation, Lancaster preprint no. LANCS-TH/9614, hep-th/960943I;

A. Linde, Prospects of inflationary cosmology, Stanford University preprint no. SU-1TP-96-43, astro$\mathrm{ph} / 9610077$.

15| P. Morse and H. Feshbach, Methods of Theoretical Physics, Vol. 1 (McGraw-Hill, New York, 1953).

161 S.W. Hawking, Nucl. Phys. B 239 (1984) 257;

D.N. Page, Hawking's wave function of the universe, in Quantum Concepts in Space and Time, ed. R. Penrose and C.J. Isham (Clarendon, Oxford, 1986).

$17 \mid$ C. Kiefer, Phys. Rev. D 38 (1988) 1761.

181 H.-D. Conradi, Phys. Rev. D 46 (1992) 612.

19| S.W. Hawking and D.N. Page, Phys. Rev. D 42 (1990) 2655.

$20 \mid$ S.P. Kim. Phys. Rev. D 46 (1992) 3403.

211 A. Mostafazadeh, Two-component formulation of the Wheeler-DeWitt equation, University of Alberta preprint no. Thy-36-96, gr-qc/9610012.

$22 \mid$ D.J. Kaup and A.P. Vitello, Phys. Rev. D 9 (1974) 1648. 